\title{
Nonlinear Perron-Frobenius theory and discrete event systems
}

\author{
Stéphane Gaubert \\ INRIA \\ Domaine de Voluceau \\ 78153 Le Chesnay Cédex \\ France \\ Stephane.Gaubert@inria.fr
}

ABSTRACT. We show how methods from nonlinear spectral theory can be used to analyse the time behaviour of dynamical discrete event systems.

RÉSUMÉ. Nous montrons comment analyser le comportement temporel des systèmes à événements discrets à l'aide de résultats de théorie spectrale non-linéaire.

KEYWORDS: monotone maps, nonlinear spectral theory, discrete event systems, repeated games. MOTS-CLÉS : applications monotones, théorie spectrale non-linéaire, systèmes à événements discrets, jeux répétés.

RS - JESA - 39/2005. MSR'05, pages 175 à 190 


\section{Introduction}

This survey paper concerns the application of non-linear Perron-Frobenius theory to the analysis of discrete event systems.

Discrete event systems can often be modelled by a dynamics of the form

$$
x(k)=f(x(k-1))
$$

where $f$ is a monotone self-map of a subset of $\mathbb{R}^{n}$. The coordinates of the vector $x(k)$ yield quantitative measures of performance: dates of $k$-th occurrence of certain repetitive events, quantities of parts of certain kinds produced up to time $k$, etc. Monotonicity is a natural assumption: it means that the earlier a job is started, the earlier it is finished.

Questions of interest include computing fixed points, sometimes in a generalised sense, and determining the asymptotic behaviour of orbits. For instance, one may expect every coordinate of $x(k)$ to growth arithmetically as $k$ tends to infinity. Arithmetical growth rate give performance measures called throughput or cycle time. One may also ask whether $x(k)$ will approach or reach a periodic orbit, perhaps after a suitable normalisation, or one may look for an initial condition $x(0)$ whose orbit is stationary, in a suitable sense.

Nonlinear Perron-Frobenius theory gives methods to address these questions. It deals with the nonlinear extensions of the spectral theory of linear monotone maps. It has been developed by many researchers and schools, and arises in several fields, such as control and game theory, linear algebra, mathematical biology, or mathematical economics. We shall not attempt to survey the field here, see (Nussbaum, 1988) and the reference therein for an overview. We shall rather emphasise aspects which seem relevant to discrete event systems, paying a special attention to related problems in optimal control and game theory.

Several authors have developed monotone dynamical models of timed discrete event systems, in order to generalise the min-plus or max-plus linear representations of timed event graphs. The present approach grew out, in particular, after (Olsder, 1991), (Gunawardena, 1994), (Vincent, 1997), (Cohen, Gaubert and Quadrat, 1995, 1997). This survey is based specially on (Gaubert and Gunawardena, 1998b, 2004), (CochetTerrasson, Gaubert and Gunawardena, 1999), (Akian and Gaubert, 2003). We could not list all the relevant works, due to the short space, so the reader is invited to look at the references of our references.

\section{General definitions and basic properties}

\subsection{Monotonicity, homogeneity, and nonexpansiveness}

We say that a self-map $f$ or $\mathbb{R}^{n}$ is monotone if

$$
x \leq y \Rightarrow f(x) \leq f(y),
$$


where $\leq$ denotes the standard ordering of $\mathbb{R}^{n}$. We say that $f$ is additively homogeneous if it commutes with the addition of a constant, meaning that

$$
f(\lambda+x)=\lambda+f(x),
$$

for all $x \in \mathbb{R}^{n}$ and $\lambda \in \mathbb{R}$, where $\lambda+x:=\left(\lambda+x_{1}, \ldots, \lambda+x_{n}\right)$. We say that $f$ is additively subhomogeneous if $f(\lambda+x) \leq \lambda+f(x)$, for all $x \in \mathbb{R}^{n}$ and $\lambda \in \mathbb{R}_{+}$, where $\mathbb{R}_{+}$denotes the set of nonnegative real numbers. Monotone (sub)homogeneous maps are nonlinear generalisations of (sub)stochastic matrices.

Additive homogeneity is a natural assumption for discrete event systems: if the entries of the vector $x(k)$ in [1] represent dates, it means that if we delay of $\lambda$ time units the initial condition, the whole trajectory will be delayed of $\lambda$ time units.

We say that a map $f$ is nonexpansive in a metric $d$ if $d(f(x), f(y)) \leq d(x, y)$. In particular, $f$ is nonexpansive in the sup-norm if

$$
\|f(x)-f(y)\|_{\infty} \leq\|x-y\|_{\infty} .
$$

Crandall and Tartar (1980) observed that an additively homogeneous map is monotone if and only if it is nonexpansive in the sup-norm. It is also known that a monotone map is nonexpansive in the sup-norm if and only if it is subhomogeneous, see for instance (Akian et al., 2004, Lemma 3.3). Monotone additively homogeneous maps have been called topical functions by Gunawardena and Keane (1995). They observed that topical functions are characterised by the property of being "nonexpansive" with respect to the map

$$
\mathrm{t}(x):=x_{1} \vee \cdots \vee x_{n},
$$

meaning that:

$$
\mathrm{t}(f(x)-f(y)) \leq \mathrm{t}(x-y) .
$$

The quantity " $\mathrm{t}(x)$ " should be read as "top of $x$ ", which explains the name "topical".

The Hilbert's seminorm is defined by

$$
\|x\|_{H}=\max _{1 \leq i \leq n} x_{i}-\min _{1 \leq i \leq n} x_{i},
$$

so that $\|x\|_{H}=0$ if and only if $x$ is a constant vector. Monotone and additively homogeneous maps are nonexpansive in Hilbert's seminorm.

A monotone additively subhomogeneous self-map $f$ of $\mathbb{R}^{n}$ can be represented by a monotone additively homogeneous self-map $g$ of $\mathbb{R}^{n+1}$ as follows:

$$
g(x, y)=(f(x-y)+y, y),
$$

where $x \in \mathbb{R}^{n}$ and $y \in \mathbb{R}$. This is analogue to adding a cemetery state to a substochastic matrix to make it stochastic. Since $g^{k}(x, 0)=\left(f^{k}(x), 0\right)$, the behaviour of $f$ can be deduced from the one of $g$. 
If $f$ is nonexpansive in any norm, we may consider the following limit, called cycle time,

$$
\chi(f)=\lim _{k \rightarrow \infty} f^{k}(x) / k,
$$

where $f^{k}$ denotes the $k$-th iterate of $f$. Since $f$ is nonexpansive, the limit, if it exists, is independent of $x$. We shall discuss further the problem of the existence of the cycle time in Section 4.2 .

We say that $u \in \mathbb{R}^{n}$ is an additive eigenvector with additive eigenvalue $\lambda \in \mathbb{R}$ of a monotone additively homogeneous self-map $f$ of $\mathbb{R}^{n}$ if

$$
f(u)=\lambda+u \text {. }
$$

Then, $f^{k}(u)=k \lambda+u$, for all $k \geq 1$. Since $f$ is nonexpansive, it follows that for all $x \in \mathbb{R}^{n}, f^{k}(x)$ stays at bounded distance from $k \lambda+u$ as $k$ tends to infinity. In particular,

$$
\chi(f)=(\lambda, \ldots, \lambda) .
$$

The existence of an additive eigenvector is a central problem: we discuss it in Section 4.1

Examples of monotone additively homogeneous maps can be easily constructed, because monotonicity and additive homogeneity are preserved by the operations of pointwise supremum, pointwise infimum, convex combination, and composition.

\subsection{The multiplicative setting}

It $f$ is a monotone self-map of $\mathbb{R}^{n}$, it is convenient to consider the conjugate map

$$
E(f)=\exp \circ f \circ \log , \mathbb{R}_{+*}^{n} \rightarrow \mathbb{R}_{+*}^{n},
$$

where $\mathbb{R}_{+*}$ denotes the set of positive real numbers, exp denotes the map from $\mathbb{R}^{n}$ to $\mathbb{R}_{+*}^{n}$ which does exp entrywise, and $\log :=\exp ^{-1}$. The conjugate $g:=E(f)$ is also monotone. When $f$ is additively homogeneous, $g$ is multiplicatively homogeneous, meaning that $g(\mu x)=\mu g(x)$, for all positive scalars $\mu$. Similarly, when $f$ is additively subhomogeneous, $g$ is multiplicatively subhomogeneous, meaning that $g(\mu x) \leq \mu g(x)$, for all scalars $\mu \geq 1$.

The sup-norm on $\mathbb{R}^{n}$ corresponds to the Thompson's part metric on $\mathbb{R}_{+*}^{n}$, $d_{T}(x, y)=\|\log x-\log y\|_{\infty}$, and Hilbert's seminorm corresponds to the Hilbert's projective metric, $d_{H}(x, y)=\|\log x-\log y\|_{H}$. This is a projective metric, since $d_{H}(x, y)=0$ if and only if the vectors $x$ and $y$ are scalar multiples. Nonexpansiveness properties of $f$ in terms of the sup-norm or of Hilbert's seminorm translate to nonexpansiveness properties of $g$ in terms of Thompson's part metric, or Hilbert's projective metric, respectively. The multiplicative cycle time of $g, \vartheta(g) \in \mathbb{R}_{+*}^{n}$, measures the geometric growth rate of the orbits,

$$
\vartheta_{i}(g)=\lim _{k \rightarrow \infty}\left(f_{i}^{k}(x)\right)^{1 / k}
$$


Of course, $\vartheta(g)=\exp (\chi(f))$, and the existence of $\chi(f)$ is equivalent to the existence of $\vartheta(g)$. We shall see in Section 4 that by considering $f$ and $E(f)$, we get two points of views on the same dynamics, bringing complementary informations.

\section{A guided tour of monotone homogeneous maps}

\subsection{Max-plus linear maps, min-plus linear maps, and timed event graphs}

The coordinates of a max-plus linear map $f: \mathbb{R}^{p} \rightarrow \mathbb{R}^{n}$ are given by:

$$
f_{i}(x)=\max _{1 \leq j \leq p} F_{i j}+x_{j}, \text { for } 1 \leq i \leq n,
$$

with $F_{i j} \in \mathbb{R} \cup\{-\infty\}$. If every row of the matrix $\left(F_{i j}\right)$ has at least one finite entry, the map $f$ sends $\mathbb{R}^{p}$ to $\mathbb{R}^{n}$. Obviously, $f$ is monotone and additively homogeneous. The dater functions of timed event graphs, firing at the earliest, are given by a dynamics of the form $x(k)=f(x(k-1))$, where $f$ is a max-plus linear self-map of $\mathbb{R}^{n}$. See (Baccelli et al., 1992) for more background.

We say that a map $f$ is min-plus linear if the map $x \mapsto-f(-x)$ is max-plus linear. The counter functions of timed event graphs, firing at the earliest, are governed by a dynamics of the form $x(k)=f(x(k-1))$, where $f$ is min-plus linear (Baccelli et al., 1992).

\subsection{Monotone linear maps and their conjugates}

If $M$ is a $n \times n$ nonnegative matrix with no zero row, then, the map

$$
g(x)=M x,
$$

is a monotone and multiplicatively homogeneous self-map of $\mathbb{R}_{+*}^{n}$. Max-plus (or minplus) linear maps arise as limits of suitably scaled conjugates of such maps. Indeed, $f=E(g)$ is given by

$$
f_{i}(x)=\log \sum_{1 \leq j \leq n} \exp \left(\log M_{i j}+x_{j}\right)
$$

$$
\text { and } \lim _{\beta \rightarrow \infty} \beta^{-1} \log \sum_{1 \leq j \leq n} \exp \left(\beta\left(\log M_{i j}+x_{j}\right)\right)=\max _{1 \leq j \leq p} \log M_{i j}+x_{j} \text {. }
$$

\subsection{Convex monotone additively subhomogeneous maps and stochastic control}

Dynamic programming operators of stochastic control problems with state space $\{1, \ldots, n\}$ and action space $A$ can be written as

$$
f(x)=\sup _{a \in A} P^{a} x+r^{a} .
$$


For all $a \in A, P^{a}$ is a $n \times n$ substochastic matrix ( $P_{i j}^{a}$ gives the probability of transition from $i$ to $j$ when action $a$ is chosen), and $r^{a}$ is a vector ( $r_{i}^{a}$ gives the reward received in state $i$, when action $a$ is chosen). The set of actions $A$ is possibly infinite, but we require $\sup _{a \in A} r^{a}$ to be finite, so that $f$ takes finite values.

Since the matrices $P^{a}$ are nonnegative, the map $f$ is monotone. It is also convex, meaning that its coordinates are convex functions. Using Legendre-Fenchel duality, one can show that any monotone additively subhomogeneous convex function can be written as [3], see (Akian and Gaubert, 2003). A remarkable case arises when $f$ is polyhedral, meaning that $\mathbb{R}^{n}$ can be covered by finitely many polyedra on which $f$ is affine. Then, the set $A$ can be chosen to be finite.

The map $f$ in [2] is monotone, additively homogeneous, and convex. A way to see this is to write it explicitly as a supremum of affine maps:

$$
f_{i}(x)=\sup _{p \in \Sigma_{n}} p \cdot x+p \cdot \log M_{i \cdot}-S(p),
$$

where $\Sigma_{n}:=\left\{x \in \mathbb{R}_{+}^{n} \mid x_{1}+\cdots+x_{n}=1\right\}$ is the simplex, $M_{i}$. denotes the $i$-th row of $M$, and $S(p)=p_{1} \log p_{1}+\cdots+p_{n} \log p_{n}$ is the entropy function. More generally, if $\left(M^{a}\right)_{a \in A}$ is a family of nonnegative matrices, and if the matrix $\sup _{a \in A} M^{a}$ is finite and has no zero row, then, the map

$$
g(x)=\sup _{a \in A} M^{a} x
$$

is such that $E^{-1}(g)$ is monotone, additively homogeneous, and convex.

\subsection{Concave monotone maps leaving the nonnegative cone invariant and fluid approximation of time Petri nets}

Maps of the form

$$
g(x)=\inf _{a \in A} M^{a} x+c^{a},
$$

where the $M^{a}$ are nonnegative matrices, and the $c^{a}$ are nonnegative vectors, arise from the following fluid approximation of timed Petri nets (Cohen, Gaubert and Quadrat, 1995, 1998).

Consider a timed Petri net, with transitions $q_{1}, \ldots, q_{n}$ and places $p_{1}, \ldots, p_{r}$. We denote by $\mu_{i j}$ the number of arcs from place $p_{j}$ to transition $q_{i}$, and by $\mu_{j i}$ the number of arcs from transition $q_{i}$ to place $p_{j}$. We denote by $m_{j}$ the initial marking of place $p_{j}$, and by $\tau_{j}$ the holding time of this place. In order to write a fluid approximation, we assume that for every place $p_{j}$, some numbers $\nu_{i j} \geq 0$ are given: a proportion $\nu_{i j}$ of the tokens of place $p_{j}$ is assigned to transition $q_{i}$. We denote by $x_{i}(t)$ the cumulated number of firings of transition $q_{i}$ up to time $t$, and by $z_{j}(t)$ the cumulated number of 
tokens that have completed their sojourn time in place $p_{j}$ before or at time $t$. The fluid behaviour is represented by the following dynamics

$$
x_{i}(t)=\min _{j \rightarrow i} \mu_{i j}^{-1} \nu_{i j} z_{j}(t), \quad z_{j}(t)=m_{j}+\sum_{k \rightarrow j} \mu_{j k} x_{k}\left(t-\tau_{j}\right) .
$$

By writing " $j \rightarrow i$ " in the first equation, we mean that the minimum is taken over the indices $j$ such that the place $p_{j}$ is a predecessor of transition $q_{i}$. Similarly, the sum is taken over all indices $k$ such that the transition $q_{k}$ is a predecessor of place $p_{j}$.

If $\tau_{j}=1$ for all places $p_{j}$, by eliminating the variables $z_{j}$, we get $x(t)=g(x(t-$ 1)) where $g$ is of the form [5]. The case of integer holding times can be treated along the same lines, provided that there is no circuit consisting only of places with zero holding time.

The following example of timed Petri net, together with its fluid approximation, is taken from (Cohen, Gaubert and Quadrat, 1995). The routing proportions are shown on the arcs: for instance, a proportion $\beta$ of the tokens of place $p_{2}$ are reserved for the firing of transition $q_{2}$.

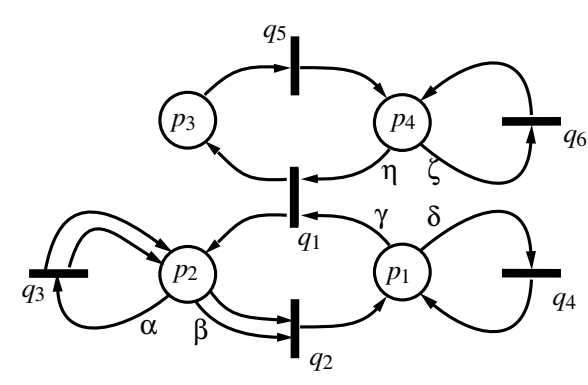

$$
\begin{aligned}
& x_{1}(t)=\min \left(\gamma z_{1}(t), \eta z_{4}(t)\right) \\
& x_{2}(t)=\frac{\beta}{2} z_{2}(t) \\
& x_{3}(t)=\alpha z_{2}(t) \\
& x_{4}(t)=\delta z_{1}(t) \\
& x_{5}(t)=z_{3}(t) \\
& x_{6}(t)=\zeta z_{4}(t) \\
& z_{1}(t)=m_{1}+x_{2}(t-1)+x_{4}(t-1) \\
& z_{2}(t)=m_{2}+2 x_{3}(t-1)+x_{1}(t-1) \\
& z_{3}(t)=m_{3}+x_{1}(t-1) \\
& z_{4}(t)=m_{4}+x_{5}(t-1)+x_{6}(t-1)
\end{aligned}
$$

\subsection{Min-max functions and beyond}

Min-max functions have been introduced by Olsder, (1991) and Gunawardena, (1994). They are obtained by composing max-plus linear and min-plus linear maps. They occur as dynamic programming operators of deterministic zero-sum two-players games, like the following. Consider a digraph with set of nodes $\{1, \ldots, n\}$, and with set of $\operatorname{arcs} E$. To every $\operatorname{arc}(i, j) \in E$ is attached a payoff, $C_{i j} \in \mathbb{R}$. Two players, call "Max" and "Min", will move alternatively a token on this graph. A turn consists of two moves, by "Max and "Min", successively. If the token is in node $i$, it must be moved to a node $j$ such that there is an arc from $i$ to $j$. At every move from $i$ to $j$, "Min" pays to "Max" the amount $C_{i j}$. If the two players agree to play exactly $k$ turns, and if the initial position of the token is $i$, the value of the player "Max" (which is the same as the opposite of the value of the player "Min") is given by the $i$-th entry of $f^{k}(0)$, where

$$
f_{i}(x)=\max _{\substack{1 \leq j \leq n \\(i, j) \in E}} C_{i j}+\min _{\substack{1 \leq k \leq n \\(j, k) \in E}} C_{j k}+x_{k}
$$


We next give an example of graph, together with two coordinates of the map $f$ :

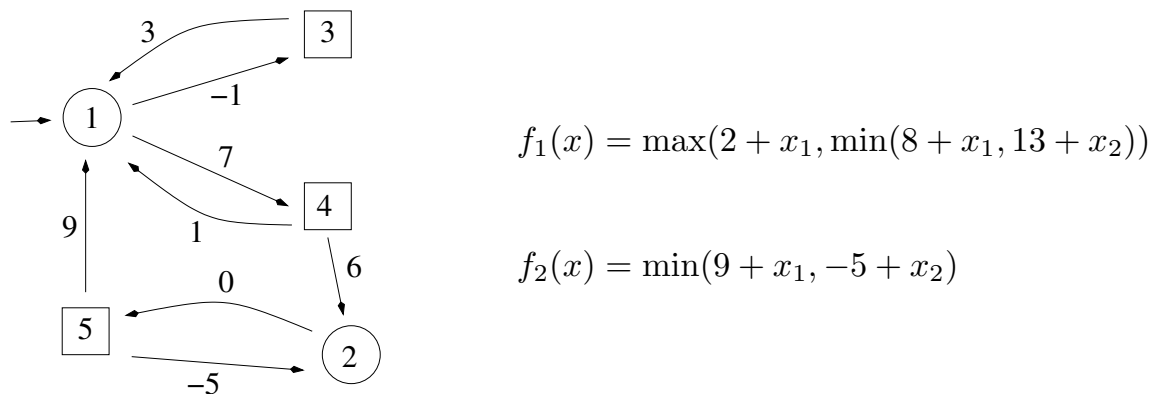

The circles represent nodes at which "Max" plays, and the squares represent nodes at which "Min" plays. The initial node, labelled "1", is shown by an input arrow.

Min-max functions arise when modelling limited synchronisation phenomena: for instance, a professor have $n$ students arriving at times $x_{1}, \ldots, x_{n}$ may wait for the first $q$ students among $n$ to be present to start his lecture. Then, the lecture will start at time

$$
\min _{\substack{I \subset\{1, \ldots, n\} \\|I|=q}} \max _{i \in I} x_{i}
$$

where $|I|$ denotes the cardinality of the set $I$.

Min-max functions are a tool. For instance, the general system of max-plus linear equations can be written as

$$
\max _{1 \leq j \leq n} A_{i j}+x_{j}=\max _{1 \leq j \leq n} B_{i j}+x_{j}, \text { for } 1 \leq i \leq p
$$

where the $A_{i j}, B_{i j}$ are given numbers in $\mathbb{R} \cup\{-\infty\}$. This is equivalent to the system of inequalities $x_{j} \leq-A_{i j}+\max _{1 \leq k \leq n} B_{i k}+x_{k}$, when $A_{i j} \neq-\infty$, and $x_{j} \leq-B_{i j}+\max _{1 \leq k \leq n} A_{i k}+x_{k}$, when $B_{i j} \neq-\infty$. Except in trivial cases, this can be rewritten as $x \leq f(x)$ for some min-max function $f$. Some problems of feedback synthesis for timed event graphs reduce to systems of equations of this kind (Katz, 2005). Min-max functions also arise in certain discretisation schemes of Hamilton-Jacobi equations (Akian, Gaubert and Lakhoua, 2004a), and in static analysis of programs (Costan et al., 2005).

Min-max functions play a remarkable role in the class of monotone additively homogeneous maps. An observation of Gunawardena and Sparrow (Gunawardena, 2003), and Rubinov and Singer (2001), shows that any monotone additively homogeneous map $f$ can be written as an (infinite) supremum of min-plus linear maps, $f(x)=\sup _{y \in \mathbb{R}^{n}} \min _{1 \leq j \leq n}\left(x_{j}-y_{j}\right)+f(y)$. This implies that any monotone additively homogeneous map can be uniformly approximated by min-max functions on compact sets. This also implies that any periodic orbit of a monotone additively homogeneous map is a periodic orbit of a min-max function. 
Min-max functions can be obtained as limits from a family of maps arising in the study of matrix scaling problems (Menon and Schneider, 1969), by a deformation similar to the one shown in Section 3.2.

To complete this guided tour of monotone homogeneous maps, let us mention that stochastic repeated games have dynamic programming operators of the form:

$$
f(x)=\sup _{a \in A} \inf _{b \in B} P^{a b} x+r^{a b},
$$

where the $P^{a b}$ are nonnegative matrices, and the $r^{a b}$ are vectors, corresponding to the actions $a$ and $b$ of the two players.

\section{Spectral theory of monotone homogeneous maps}

\subsection{Existence of an additive eigenvector}

If $f$ is nonexpansive in some norm and has a fixed point, every orbit of $f$ must be bounded. A theorem of Nussbaum, stated in the more general context of cones of Banach spaces, provides a converse.

Theorem 4.1 (See Th. 4.1 of Nussbaum, (1988)). A self map of $\mathbb{R}^{n}$ that is nonexpansive in some norm has a fixed point if and only if it has one bounded orbit.

Nussbaum proved this by constructing, from the $\omega$-limit set of one orbit, a compact convex set that is invariant by the map, to which he applied Leray-Schauder's fixed point theorem. Let us now apply Nussbaum's theorem to show the existence of additive eigenvectors. If $u, v \in \mathbb{R}^{n}$, we set $u \sim v$ if $u=\lambda+v$ for some $\lambda \in \mathbb{R}$. The quotient $\mathbb{R}^{n} / \sim$ is a vector space, which is a normed by Hilbert's seminorm. A monotone and additively homogeneous self-map $f$ or $\mathbb{R}^{n}$ induces a self-map of $\mathbb{R}^{n} / \sim$ which is nonexpansive in Hilbert's seminorm, and $f$ has an eigenvector if and only if its quotient map has a fixed point. It remains to find invariant sets that are bounded in Hilbert's seminorm. Natural invariant sets are the slices of $f$, which are of the form $S_{\mu}^{\lambda}(f)=\left\{x \in \mathbb{R}^{n} \mid \mu+x \leq f(x) \leq \lambda+x\right\}$, for some $\lambda \in \mathbb{R} \cup\{+\infty\}$ and $\mu \in \mathbb{R} \cup\{-\infty\}$. We get as a corollary of Theorem 4.1 .

Corollary 4.1. If $f$ is a monotone additively homogeneous self-map of $\mathbb{R}^{n}$ and if some slice $S_{\mu}^{\lambda}$ is non-empty and bounded in Hilbert's seminorm, then, $f$ admits an additive eigenvector.

Checking whether a particular slice $S_{\mu}^{\lambda}$ is bounded in Hilbert's seminorm can be difficult. However, there are general methods to check whether all slices are bounded. We call recession function of $f$ the map $\hat{f}(x):=\lim _{\nu \rightarrow \infty} \nu^{-1} f(\nu x)$.

Theorem 4.2 (Th. 13 of Gaubert and Gunawardena (2004)). Assume that $f$ is a monotone additively homogeneous self-map of $\mathbb{R}^{n}$ whose recession function $\hat{f}$ exists and has only fixed points on the diagonal. Then, all the slices of $f$ are bounded in Hilbert's seminorm. In particular, $f$ has an additive eigenvector. 
There is an even simpler condition involving the additive super-eigenspace, $S^{\lambda}:=$ $S_{-\infty}^{\lambda}=\left\{x \in \mathbb{R}^{n} \mid f(x) \leq \lambda+x\right\}$. Let $G(f)$ denote the digraph with nodes $\{1, \ldots, n\}$, such that there is an arc from $i$ to $j$ if $\lim _{\nu \rightarrow+\infty} f_{i}\left(\nu e_{j}\right)=+\infty$, where $e_{j}$ denote the $j$-th vector of the canonical basis of $\mathbb{R}^{n}$.

Theorem 4.3 (Th. 10 of Gaubert and Gunawardena (2004)). Let $f$ be a monotone additively homogeneous self-map of $\mathbb{R}^{n}$. If $G(f)$ is strongly connected, then all the super-eigenspaces $S^{\lambda}$ are bounded in Hilbert's seminorm. In particular, $f$ has an additive eigenvector.

A characterisation of the $f$ for which all the super-eigenspaces are bounded in Hilbert's seminorm is also given, in terms of aggregated graphs. A characterisation of the boundedness of all the slices is lacking.

\subsection{Some results concerning the cycle time}

The top cycle time, $\bar{\chi}(f)$, is defined by $\bar{\chi}(f)=\lim _{k \rightarrow \infty} \mathrm{t}\left(f^{k}(x)\right) / k$. A subadditive argument shows that:

Proposition 4.1 (Vincent (1997), Gunawardena and Keane (1995)). The top cycle time of a monotone additively homogeneous self-map of $\mathbb{R}^{n}$ always exists.

There is always one coordinate which realises the top cycle time:

Theorem 4.4 (Th. 8 of Gaubert and Gunawardena (2004)). Let $f$ be a monotone additively homogeneous self-map of $\mathbb{R}^{n}$, and let $x \in \mathbb{R}^{n}$. There exists $1 \leq i \leq n$, such that, for all $k \in \mathbb{N}, f_{i}^{k}(x) \geq x_{i}+k \bar{\chi}(f)$.

For nonexpansive self-maps of Banach spaces, the existence of the cycle time is related to the strict convexity of the balls (Kohlberg and Neyman, 1981). In particular, a self-map of $\mathbb{R}^{2}$ that is nonexpansive in the sup-norm need not have a cycle time (Kohlberg and Neyman, 1981), and a monotone additively homogeneous selfmap of $\mathbb{R}^{3}$ need not have a cycle time (Gunawardena and Keane, 1995).

A useful notion in the study of the cycle time problem is the following. If $v, \eta \in$ $\mathbb{R}^{n}$, we say that $t \mapsto v+t \eta$ is an invariant half-line of $f$ if $f(v+t \eta)=v+(t+1) \eta$ for all $t>0$.

Theorem 4.5 (Kohlberg (1980)). If $f$ is a polyhedral self-map of $\mathbb{R}^{n}$ that is nonexpansive in some norm, then $f$ has an invariant half-line. In particular, $\chi(f)$ exists.

Bewley and Kohlberg (1976) showed that dynamic programming operators of certain stochastic games with finite state and action spaces have a cycle time, because the discounted value $\xi_{\alpha} \in \mathbb{R}^{n}$, solution of $f\left(\alpha \xi_{\alpha}\right)=\xi_{\alpha}$, for $0 \leq \alpha<1$, has a Puiseux series expansion in $\alpha$. More generally, 
Theorem 4.6 (Neyman (2003)). A semi-algebraic self-map of $\mathbb{R}^{n}$ that is nonexpansive in some norm has a cycle time.

Neyman showed that if $(1-\alpha) \xi_{\alpha}$ has bounded variation as $\alpha$ tends to $1^{-}$, then $\chi(f)=\lim _{\alpha \rightarrow 1^{-}}(1-\alpha) \xi_{\alpha}$. Other results, also exploiting vanishing discount, appeared in (Rosenberg and Sorin, 2001). A different method relies on the observation that the set of self-maps of $\mathbb{R}^{n}$ that are non-expansive in a given norm is closed for the topology of uniform convergence on $\mathbb{R}^{n}$ (Gaubert and Gunawardena, 2000), see also (Bousch and Mairesse, 2003), where a related idea is used. As an application, let us denote by $\mathscr{H}$ the set of monotone additively subhomogeneous self-maps of $\mathbb{R}^{n}$, whose coordinates can be written as terms in the grammar:

$$
\begin{array}{r}
X \rightarrow 0, x_{1}, \ldots, x_{n}, \lambda+X, \max (X, X), \min (X, X), \alpha X+(1-\alpha) X, \\
\beta^{-1} \log (\exp (\beta X)+\exp (\beta X)),
\end{array}
$$

where $\lambda \in \mathbb{R}, 0 \leq \alpha \leq 1$, and $\beta \in \mathbb{R} \backslash\{0\}$. For instance, the term $\min \left(9,7+\log \left(\exp \left(x_{1}\right)+\exp \left(\max \left(x_{2},\left(x_{3}+x_{1}\right) / 2\right)\right)\right)\right)$ is produced by this grammar. Up to a trivial generalisation, the class $\mathscr{H}$ is equivalent to some classes considered in (Nussbaum, 1988), (Gaubert and Gunawardena, 1998), and (Gunawardena, 2003). All the examples of monotone additively subhomogeneous maps presented in this paper belong to the class $\mathscr{H}$. One can check that every map $f \in \mathscr{H}$ can be uniformly approximated by polyhedral monotone additively homogeneous self-maps of $\mathbb{R}^{n}$ (it suffices to check this property for atomic expressions). It follows that:

Corollary 4.2 (Gaubert and Gunawardena (2000)). Every map in $\mathscr{H}$ admits a cycle time.

The map $g$ governing the fluid approximation of timed Petri nets can be written as [5], where the set $A$ is finite. Since $E^{-1}(g)$ belongs to $\mathscr{H}$, we deduce that:

Corollary 4.3 (Existence of the growth rate of fluid Petri nets). If $x(t)$ denotes the counter function of the fluid approximation of a timed Petri net, governed by the dynamics [6], if all the holding times are integers, and if there is no circuit with zero holding time, then, the limits

$$
\lim _{t \rightarrow \infty}\left(x_{i}(t)\right)^{1 / t}, i=1, \ldots, n
$$

exist, and are independent of the initial conditions, provided these are positive.

Cohen, Gaubert and Quadrat (1995) showed that for a subclass of fluid Petri nets, called undiscounted, and characterised by the existence of certain invariants, the limits $\lim _{t \rightarrow \infty} x_{i}(t) / t$ does exist.

\subsection{Eigenvectors in the closed cone}

If $f$ is a monotone additively homogeneous self-map of $\mathbb{R}^{n}$, we introduced in Section 2.2 the conjugate $g=E(f)$, which is monotone and multiplicatively homo- 
geneous. The additive eigenproblem for $f$ corresponds to the multiplicative eigenproblem for $g$, consisting in finding $v \in \mathbb{R}_{+*}^{n}$ and $\mu \in \mathbb{R}_{+*}$ such that

$$
g(v)=\mu v .
$$

The multiplicative eigenproblem is better posed if we allow $v$ to be a non-zero vector in the closed cone, $\mathbb{R}_{+}^{n}$. This is licit because $g$ extends continuously to $\mathbb{R}_{+}^{n}$ (Burbanks, Nussbaum and Sparrow, 2003). By applying Brouwer's fixed point theorem to the map $x \mapsto g(x) /\left(\sum_{i} g_{i}(x)\right)$ which is well defined and preserves the simplex (except in the trivial case when $g(x)=0$ for some non-zero $x \in \mathbb{R}_{+}^{n}$ ), we get that $g$ has always at least one eigenvector. We call support of an eigenvector $v$ the set $S(v):=\{1 \leq$ $\left.i \leq n \mid v_{i} \neq 0\right\}$. If a nonnegative vector $x$ has the same support as the eigenvector $v$, we can write $\alpha v \leq x \leq \beta v$, for some $\alpha, \beta>0$, and so

$$
\alpha \mu^{k} v \leq g^{k}(x) \leq \beta \mu^{k} v, \text { for all } k \geq 1 .
$$

We deduce that

$$
\lim _{k \rightarrow \infty}\left(g_{i}^{k}(x)\right)^{1 / k}=\mu, \forall i \in S(v) .
$$

Thus, the eigenvalue $\mu$ determines the geometric growth rate of all the orbits starting from a point which has the same support as the eigenvector $v$. In particular, the number of eigenvalues, which cannot exceed the number of supports of non-zero vectors, is bounded by $2^{n}-1$. We call spectral radius of $g$ its maximal eigenvalue, and we denote it by $\rho(g)$. A result of Nussbaum, stated more generally for self-maps of finite dimensional cones, yields the following.

Proposition 4.2 (Nussbaum (1986)). The spectral radius of a continuous monotone multiplicatively homogeneous self-map $g$ of $\mathbb{R}_{+}^{n}$ coincides with

$$
\inf _{u \in \mathbb{R}_{+*}^{n}} \max _{1 \leq i \leq n} \frac{g_{i}(u)}{u_{i}}=\inf \left\{\mu>0 \mid g(u) \leq \mu u \text { for some } u \in \mathbb{R}_{+*}^{n}\right\} .
$$

The function $u \mapsto \max _{1 \leq i \leq n} \frac{g_{i}(u)}{u_{i}}$ is sometimes called the Collatz-Wielandt function, it is used to provide a short proof of the Perron-Frobenius theorem. One can deduce from the previous proposition, or show directly (Gaubert and Gunawardena, 2004), that

$$
\bar{\chi}(f)=\inf \left\{\lambda \in \mathbb{R} \mid \exists v \in \mathbb{R}^{n}, f(v) \leq \lambda+v\right\} .
$$

This characterisation is particularly useful when $f$ is convex. It allows one to compute $\bar{\chi}(f)$ as the value of a convex program. It also allows one to solve non-linear eigenvalue optimisation problems. Indeed, if $\left(f_{a}\right)_{a \in A}$ is a family of monotone additively homogeneous maps, if the set $A$ is convex, and if the map $(a, x) \mapsto f_{a}(x)$ is convex, it follows from [9] the problem of finding $a \in A$ minimising $\bar{\chi}\left(f_{a}\right)$ reduces to the following convex programming problem: minimise $\lambda$ over the set of $(\lambda, a, v) \in \mathbb{R} \times A \times \mathbb{R}^{n}$ such that $f_{a}(v) \leq \lambda+v$. This idea was used in (Gaubert, 1995) to show that the problem of minimising a linear function of the initial marking of a timed event graph (the cost of the resources), in order to guarantee a given throughput, reduces to a mixted linear program. 


\subsection{Cyclicity theorems}

If $f$ is a self-map of $\mathbb{R}^{n}$ that is nonexpansive in a polyhedral norm, then, it is known that every bounded orbit of $f$ converges to a periodic orbit, whose length can be bounded by a function depending only of the norm (and of course of the integer $n$ ), see (Nussbaum, 1990) and (Sine, 1990). In the case of the sup-norm, Nussbaum, (1990) has conjectured that the optimal upper bound is $2^{n}$. For monotone additively subhomogeneous self-maps of $\mathbb{R}^{n}$, Lemmens and Scheutzow (2003) have confirmed a conjecture of Gunawardena and Sparrow, by showing that the optimal bound is $\left(\begin{array}{c}n \\ \lfloor n / 2\rfloor\end{array}\right)$, the maximal cardinality of an antichain in $\{0,1\}^{n}$.

It follows that if $f$ is a monotone additively homogeneous map with additive eigenvalue $\lambda$, for all $x \in \mathbb{R}^{n}$, there exists an integer $c \leq\left(\begin{array}{c}n \\ \lfloor n / 2\rfloor\end{array}\right)$ such that the sequence $f^{k c}(c)-k c \lambda$ converges as $k$ tends to infinity. When $f$ is a min-max function, the limit is attained in finite time (Olsder and Perennes, 1997).

When $f$ is monotone, additively subhomogeneous, and convex, the structure of the periodic orbits of $f$ and of its eigenspace is relatively well understood, particularly when $f$ is polyhedral, (Akian and Gaubert, 2003).

The behaviour on the closed cone of the conjugate $E^{-1}(f)$ is somehow more complex: any bounded orbit of a continuous monotone multiplicatively homogeneous self-map of $\mathbb{R}_{+}^{n}$ still converges to a periodic orbit, but its length is now at most $n ! /\left(\left\lfloor\frac{n}{3}\right\rfloor !\left\lfloor\frac{n+1}{3}\right\rfloor !\left\lfloor\frac{n+2}{3}\right\rfloor !\right)$, see (Akian et al., 2004).

\subsection{Algorithmic issues}

If $f$ is monotone and additively subhomogeneous, we wish to find a fixed point $v \in \mathbb{R}^{n}$, or an additive eigenvector $v \in \mathbb{R}^{n}$ and its eigenvalue $\lambda \in \mathbb{R}$, or an invariant half line $t \mapsto v+t \eta$, where $v, \eta \in \mathbb{R}^{n}$. Such quantities allow one to determine the cycle time $\chi(f)$. When $f(x)=P x+c$ is affine, an invariant half-line is characterised by the system of equations $P \eta=\eta$ and $P v+c=v+\eta$, which is well known in potential theory.

When $f$ is a stochastic control operator like [3], with a finite action space $A$, a Newton-type algorithm, called policy iteration, initially developed by Howard, is experimentally efficient. Invariant half-lines can be computed by the multichain policy iteration algorithm of Denardo and Fox (1968).

We now present a generalisation of Howard's policy iteration, which applies to games. We assume that

$$
f=\inf _{a \in A} f_{a}
$$

where $\left(f_{a}\right)_{a \in A}$ is a finite family of monotone homogeneous self-maps of $\mathbb{R}^{n}$, and that the family $\left(f_{a}\right)_{a \in A}$ has a lower selection, meaning that for all $x \in \mathbb{R}^{n}$, there exists 
$a \in A$ such that $f(x)=f_{a}(x)$. This assumption is satisfied in game problems if we take for $A$ the set of pure strategies of one player (then, the map $f_{a}$, which is usually of the form [3], possibly with a different set of actions $A$, models the reply of the other player). The fact that the cardinality of $A$ may be large is not an obstacle, it suffices to have an efficient oracle returning the map $f_{a}$ such that $f(x)=f_{a}(x)$. We have the following general method to find a fixed point of $f$.

\section{Algorithm 4.1 (Basic policy iteration algorithm).}

1) Initialisation. Set $k=1$ and select any action $a^{1} \in A$.

2) Value determination. Compute a fixed point $x^{k}$ of $f_{a^{k}}$.

3) Compute $f\left(x^{k}\right)$.

4) If $f\left(x^{k}\right)=x^{k}$, return $x^{k}$.

5) Policy improvement. Take $a^{k+1}$ such that $f\left(x^{k}\right)=f_{a^{k+1}}\left(x^{k}\right)$. Increment $k$ and goto Step 2 .

If every map $f_{a}$ has only one fixed point, and if the maps $f_{a}$ are monotone and additively subhomogeneous, one can show that the sequence $x^{k}$ is strictly decreasing (Cochet-Terrasson, Gaubert and Gunawardena, 2001), (Costan et al., 2005). Hence, the algorithm converges in finite time. The algorithm converges if, more generally, every map $f_{a}$ has a smallest fixed point, and if this fixed point is chosen at every iteration.

More refined policy iteration algorithms allow one to solve the additive eigenproblem, and more generally, to compute an invariant half line, see (Gaubert and Gunawardena, 1998) and (Cochet-Terrasson, Gaubert and Gunawardena 1999), for the case of min-max functions, and (Cochet-Terrasson, 2001) for the more general case of polyhedral dynamic programming operators of the form [8]. Combined with the approximation method of Corollary 4.2 , this allows one to approximate the cycle time of any map in $\mathscr{H}$. Systematic tests in the special case of max-plus linear maps (CochetTerrasson et al., 1998), as well as examples of monotone fixed point equations coming from abstract interpretation (Costan et al., 2005), suggest that policy iteration is experimentally efficient, even if its theoretical complexity is still unknown. See (Zwick and Paterson, 1996) for a discussion of complexity issues concerning deterministic repeated games.

\section{References}

Akian M., Gaubert S., « Spectral Theorem for Convex Monotone Homogeneous Maps, and ergodic Control », Nonlinear Analysis. Theory, Methods \& Applications, vol. 52, n. 2, p. 637 679, 2003.

Akian M., Gaubert S., Lakhoua A., « A max-plus finite element method for solving finite horizon deterministic optimal control problems », Proceedings of MTNS'04, Louvain, Belgique, 2004. Also arXiv:math.OC/0404184 
Akian M., Gaubert S., Lemmens B., Nussbaum R., « Iteration of order preserving subhomogeneous maps on a cone », 2004. To appear in the Math. Proc. Cambridge Phil. Soc., arXiv:math.DS/0410084

Baccelli F., Cohen G., Olsder G., Quadrat J., Synchronization and Linearity, Wiley, 1992.

Bewley T., Kohlberg E., « The asymptotic solution of a recursion equation occurring in stochastic games », Math. Oper. Res., vol. 1, n. 4, p. 321-336, 1976.

Bousch T., Mairesse J., Fonctions topicales à portée finie et fonctions uniformément topicales, Préprint LIAFA n. 002, 2003.

Burbanks A. D., Nussbaum R. D., Sparrow C. T., « Extension of order-preserving maps on a cone », Proc. Roy. Soc. Edinburgh Sect. A, vol. 133, n. 1, p. 35-59, 2003.

Cochet-Terrasson J., Algorithmes d'itération sur les politiques pour les applications monotones contractantes, PhD thesis, École des Mines, 2001.

Cochet-Terrasson J., Cohen G., Gaubert S., Gettrick M. M., Quadrat J.-P., « Numerical computation of spectral elements in max-plus algebra », Proc. of the IFAC Conference on System Structure and Control, Nantes, July, 1998.

Cochet-Terrasson J., Gaubert S., Gunawardena J., « A constructive fixed point theorem for min-max functions », Dynamics and Stability of Systems, vol. 14, n. 4, p. 407-433, 1999.

Cochet-Terrasson J., Gaubert S., Gunawardena J., « Policy iteration algorithms for monotone nonexpansive maps », 2001, Privately circuled draft.

Cohen G., Gaubert S., Quadrat J., « Asymptotic Throughput of Continuous Timed Petri Nets », Proceedings of the 34th Conference on Decision and Control, New Orleans, Dec, 1995.

Cohen G., Gaubert S., Quadrat J., « Algebraic System Analysis of Timed Petri Nets », in , J. Gunawardena (ed.), Idempotency, Publications of the Isaac Newton Institute, Cambridge University Press, p. 145-170, 1998.

Costan A., Gaubert S., Goubault E., Martel M., Putot S., « A policy iteration algorithm for computing fixed points in static analysis of programs », Proceedings of CAV'05, Edimburgh, July, 2005.

Crandall M. G., Tartar L., « Some relations between non expansive and order preserving maps », Proceedings of the AMS, vol. 78, n. 3, p. 385-390, 1980.

Denardo E., Fox B., « Multichain Markov Renewal Programs », SIAM J. Appl. Math, vol. 16, n. 3, p. 468-487, 1968.

Gaubert S., « Resource Optimization and $(\mathrm{min},+)$ Spectral Theory », IEEE Trans. on Automatic Control, vol. 40, n. 11, p. 1931-1934, Nov., 1995.

Gaubert S., Gunawardena J., « The Duality Theorem for min-max functions », C. R. Acad. Sci. Paris., vol. 326, Série I, p. 43-48, 1998a.

Gaubert S., Gunawardena J., «A Non-Linear Hierarchy for Discrete Event Dynamical Systems », Proc. of the Fourth Workshop on Discrete Event Systems (WODES98), IEE, Cagliari, Italy, 1998b.

Gaubert S., Gunawardena J., « Existence of the cycle time for a class of nonexpansive maps », 2000, Privately circuled draft.

Gaubert S., Gunawardena J., « The Perron-Frobenius Theorem for Homogeneous, Monotone Functions », Trans. of AMS, vol. 356, n. 12, p. 4931-4950, 2004.

Gunawardena J., « Min-max functions », Discrete Event Dynamic Systems, vol. 4, p. 377-406, 1994. 
Gunawardena J., « From max-plus algebra to nonexpansive maps: a nonlinear theory for discrete event systems », Theoretical Computer Science, vol. 293, p. 141-167, 2003.

Gunawardena J., Keane M., On the existence of cycle times for some nonexpansive maps, Technical Report $n \hat{A}^{\circ}$ HPL-BRIMS-95-003, Hewlett-Packard Labs, 1995.

Katz R. D., « Max-plus (A,B)-invariant spaces and control of timed discrete event systems », 2005, arXiv:math.OC/0503448.

Kohlberg E., « Invariant half-lines of nonexpansive piecewise-linear transformations », Math. Oper. Res., vol. 5, n. 3, p. 366-372, 1980.

Kohlberg E., Neyman A., « Asymptotic behavior of nonexpansive mappings in normed linear spaces », Israel J. Math., vol. 38, n. 4, p. 269-275, 1981.

Lemmens B., Scheutzow M., « On the dynamics of sup-norm nonexpansive maps », 2003, to appear in Ergodic Theory Dynam. Systems.

Menon M., Schneider H., « The spectrum of an operator associated with a matrix », Linear Algebra and its Applications, vol. 2, p. 321-334, 1969.

Neyman A., « Stochastic games and nonexpansive maps », Stochastic games and applications (Stony Brook, NY, 1999), vol. 570 of NATO Sci. Ser. C Math. Phys. Sci., Kluwer Acad. Publ., Dordrecht, p. 397-415, 2003.

Nussbaum R. D., « Convexity and Log-Convexity for the Spectral Radius », Linear Algebra and its Applications, vol. 73, p. 59-122, 1986.

Nussbaum R. D., « Hilbert's projective metric and iterated nonlinear maps », Memoirs of the $A M S$, vol. 75, n. 391, 1988.

Nussbaum R. D., « Omega limit sets of nonexpansive maps: finiteness and cardinality estimates », Differential Integral Equations, vol. 3, n. 3, p. 523-540, 1990.

Olsder G., « Eigenvalues of dynamic min-max systems », J. of Discrete Event Dynamic Systems, vol. 1, p. 177-207, 1991.

Olsder G.-J., Perennes S., « Privately circuled draft », 1997.

Rosenberg D., Sorin S., « An operator approach to zero-sum repeated games », Israel J. Math., vol. 121, p. 221-246, 2001.

Rubinov A. M., Singer I., « Topical and sub-topical functions, downward sets and abstract convexity », Optimization, vol. 50, n. 5-6, p. 307-351, 2001.

Sine R., «A nonlinear Perron-Frobenius theorem », Proc. Amer. Math. Soc., vol. 109, n. 2, p. 331-336, 1990.

Vincent J., «Some ergodic results on stochastic iterative discrete events systems », DEDS: Theory and Applications, vol. 7, n. 2, p. 209-233, 1997.

Zwick U., Paterson M., « The complexity of mean payoff games on graphs », Theoret. Comput. Sci., vol. 158, n. 1-2, p. 343-359, 1996. 